\title{
Utilisation de tablettes numériques tactiles par des étudiants lors de Travaux Pratiques en Physique à l'Université
}

\section{Use of tactile digital tablets by students during practical class of Physics at the University}

\author{
Nicolas Coppens ${ }^{1 *}$ \\ ${ }^{1}$ Université de Strasbourg, INSPE, 141 avenue de Colmar, BP 40102, 67024 Strasbourg Cedex \\ ECPM, 25 rue Becquerel, 67087 Strasbourg Cedex, France.
}

\begin{abstract}
Résumé. L'utilisation des tablettes numériques tactiles devrait faciliter l'enseignement. Cependant, malgré leur introduction dans de nombreux pays, les résultats en termes d'apprentissages des élèves ou des étudiants sont assez mitigés. Pour préciser cela, nous avons étudié dans quelle mesure l'utilisation en autonomie des tablettes numériques tactiles par des étudiants pouvait les aider dans leur apprentissage lors de travaux pratiques d'électricité à l'Université. Les résultats de cette étude ont montré un impact positif: l'utilisation non prescriptive de la tablette a permis à chaque étudiant d'utiliser les fonctionnalités qui l'intéressaient et de collaborer ou demander de l'aide aux autres étudiants ou à l'enseignant s'il le souhaitait. Cela nécessite toutefois une maturité suffisante de la part des étudiants et une formation préalable aux bons usages d'un tel outil connecté, comme cela a été mis en place dans le cadre de cette étude.
\end{abstract}

\begin{abstract}
The use of tactile digital tablets in education should facilitate the learning process. However, in spite of their introduction in several countries, the results in terms of learnings aren't very good. To study it closely, we studied how the autonomous use of tactile digital tablets by students could help them in their learning during practical class of electricity at University. The results of this study showed a positive impact on the learning process. This non-prescriptive use allowed every student to use the features of the tablet which interested them and to collaborate or to ask for help when necessary. However, it requires a sufficient maturity from the students and a preliminary training in mastering the good uses of such a connected tool, such as the training session which was set up in this study.
\end{abstract}

\footnotetext{
*Corresponding author : nicolas.coppens@unistra.fr
} 


\section{Introduction}

L'utilisation des tablettes numériques tactiles dans l'enseignement peut permettre la mise en place de pratiques pédagogiques innovantes développant des modalités d'apprentissage plus collaboratives et aussi plus individualisées. Avec ces outils connectés, il est plus simple de développer une pédagogie différenciée, adaptable à des contextes d'apprentissage variés et aux besoins spécifiques de chaque apprenant, comme cela est préconisé depuis de nombreuses années par les chercheurs mais aussi par les institutions scolaires et par certaines universités [1-18]. Ces tablettes sont également conçues comme des outils de communication, favorisant le partage et les interactions entre pairs. Leur caractère individuel peut stimuler l'autonomie et la créativité des élèves. Les tablettes semblent aussi plus faciles à gérer que les ordinateurs car elles réduisent notamment un peu les cas de comportements hors tâche durant les cours, comme par exemple l'utilisation des réseaux sociaux pour un usage personnel [19]. De plus, d'après le Ministère de l'Education Nationale française [20], les tablettes « enrichissent les stratégies pédagogiques mises en œuvre par les enseignants et les apprentissages de leurs élèves. Rapidité de mise en œuvre, autonomie, légèreté, simplicité d'utilisation, mobilité sont des qualités reconnues [d'une tablette qui] peut être utilisée à tout moment pour une consultation, un exercice, un enregistrement, un travail de recherche individuel ou collectif, et cela même pour une activité de courte durée ». Ainsi, des expériences de grande ampleur sont menées pour introduire des tablettes dans l'écosystème scolaire et universitaire, soit au niveau national comme en Corée du Sud, en Turquie, en Thaïlande ou en Inde, soit au niveau des collectivités territoriales ou des établissements comme aux Etats-Unis, au Québec, au Royaume-Uni, aux Pays-Bas ou en France [21-27].

Toutefois, d'après Boujol [28], il semble que « l'intégration du numérique pour des apprentissages différenciés n'est pas ou peu été pris en compte. En fait, on peut dire de façon quelque peu caricaturale que l'enseignant se dote de moyens techniques pour améliorer ses cours mais n'intègre pas les élèves à celui-ci en tant qu'acteurs de leur propre apprentissage. [De plus,] les enseignants ont encore une très grande réticence à communiquer avec les élèves, à faire entrer le numérique dans les processus d'apprentissage, de recherche d'informations, d'interactions et dans les modalités d'échanges ». L'interprétation des résultats de PISA par l'OCDE [29] montre également que « les TIC ne sont associées à une meilleure performance des élèves que dans certains contextes, par exemple lorsque les logiciels informatiques et la connexion Internet aident à accroître le temps d'étude et les possibilités de s'exercer ». Quant à Landau [25], il ne recueille pas d'avis positif dans une enquête portant sur l'utilisation des tablettes dans une classe de Terminale, qui correspond à la dernière année avant l'Université en France. En effet, si la plupart des lycéens sont des digital natives [30], qui savent utiliser les tablettes même s'ils en ont quelquefois des usages stéréotypés, les élèves rejettent parfois cet outil car ils y voient une forme d'intrusion des adultes dans leurs usages [31] et car leur utilisation scolaire est moins riche que leur utilisation privée [32]. Une implantation réussie des tablettes semble donc notamment être celle qui permet aux élèves ou aux étudiants de bien dissocier les usages qu'ils peuvent faire avec cet objet connecté selon le contexte dans lequel ils se trouvent [22-33].

Pour vérifier cela, nous nous sommes intéressés à l'impact de l'utilisation de tablettes sur l'apprentissage dans le cadre de l'acquisition d'Ipad à partir de 2016 pour tous les étudiants et pour des enseignants de la classe préparatoire intégrée à l'Ecole Européenne de Chimie, 
de Polymères et de Matériaux (ECPM) de l'Université de Strasbourg [34]. Depuis cette date, certains enseignants de l'ECPM demandent aux étudiants une utilisation très guidée des tablettes numériques tactiles, avec des Videxos par exemple [35, 36], alors que d'autres enseignants laissent les étudiants travailler en autonomie avec leur tablette [37]. Nous avons alors étudié dans quelle mesure l'utilisation guidée puis en autonomie des tablettes numériques tactiles par ces étudiants pouvait les aider dans leur apprentissage.

\section{Méthodologie}

Pour répondre à cette question de recherche, un questionnaire a été posé en 2018 à 44 étudiants de première année universitaire et à 37 étudiants de deuxième année, après des travaux pratiques d'électricité pendant lesquels les étudiants ont utilisé les tablettes en autonomie. La passation de ce questionnaire s'est effectuée de manière anonyme pendant quarante-cinq minutes $^{\dagger}$, grâce au logiciel Socrative installé sur les tablettes. Les étudiants de première année avaient chacun à leur disposition depuis environ 6 mois un Ipad pour leurs usages universitaires mais aussi pour leurs usages personnels tandis que les étudiants de deuxième année utilisaient leur Ipad depuis plus d'un an.

Ce questionnaire comporte 57 questions à choix multiples (QCM) et 3 questions ouvertes. Pour expliquer ou mesurer l'intégration et l'impact des tablettes ainsi que pour comprendre les attitudes et les perceptions des étudiants, les questions et les items proposés dans ce questionnaire ont été rédigés à partir des observations des étudiants durant les travaux pratiques $(\mathrm{TP})$ et à partir de certains modèles théoriques. Parmi ces théories, notre attention s'est portée notamment, comme Landau [25], sur le Computer Attitude Measure for Young Students (en français, Mesure de l'Attitude envers l'Informatique pour les Jeunes Etudiants/Elèves), un instrument de mesure qui utilise l'Affect Towards Computer (Attitude envers la science informatique) [38] et le Technology Acceptance Model (Modèle d'Acceptation de la Technologie) de Davis [39], qui a travaillé sur l'acceptation de la technologie dans les écoles, ce modèle étant lui-même issu du Theory of Reasonned Action (Théorie de l'Action Raisonnée), développé par Fishbein \& Ajzen [23]. Enfin, nous nous sommes aussi appuyés sur l'évaluation des mesures d'apprentissage et des mesures d'engagement [40].

Ainsi, ce questionnaire, divisé en deux grandes parties :

- Quel(s) usage(s) avez-vous fait(s) de votre tablette durant les TP d'électricité ? pour réviser les TP d'électricité ?

- Utiliser la tablette vous a-t-il aidé lors les TP d'électricité ?

est composé par exemple des questions suivantes, parmi les 60 proposées.

\section{Lors des TP d'électricité, j'ai utilisé ma tablette pour :}

- annoter les énoncés des TP.

[] OUI [] NON [] Je ne sais plus

(les énoncés des questions et les possibilités de réponses sont en réalité plus détaillées pour chacune des questions posées )

- prendre des photographies des montages réalisés, de l'écran de l'oscilloscope...

[] OUI [] NON [] Je ne sais plus

- partager des fichiers ou des informations avec d'autres groupes de TP.

[] OUI [] NON [] Je ne sais plus

${ }^{\dagger}$ Les étudiants qui le souhaitaient pouvaient disposer de temps supplémentaire mais cela n'a été demandé par aucun étudiant. 
- me détendre pendant quelques minutes durant les TP en consultant mes mails personnels...

[] OUI [] NON [] Je ne sais plus

\section{Pour réviser, j'ai utilisé ma tablette afin de :}

- regarder les photographies prises pendant les séances de TP.

[] OUI [] NON [] Je ne sais plus

- réaliser conjointement avec mon binôme une (ou des) fiche(s) de révision.

[] OUI [] NON [] Je ne sais plus

- rechercher sur Internet des informations.

[] OUI [] NON [] Je ne sais plus

\section{Utiliser la tablette m'a aidé lors des TP d'électricité.}

En effet :

[] OUI [] NON [] Je ne sais pas

- mettre des annotations sur les photos prises pendant les TP permet de mieux comprendre.

[] OUI [] NON [] Je ne sais pas

- avec la tablette, je suis tenté d'aller sur Internet et cela me perturbe.

[] OUI [] NON [] Je ne sais pas

De plus, pour tester la cohérence des réponses sachant que le questionnaire est assez long et que certains étudiants étaient allophones, nous avons posé quelques questions « contradictoires » afin de vérifier que les réponses données à ces questions étaient bien opposées.

J'apprends mieux en relisant des documents sur la tablette plutôt que des documents écrits sur une feuille.

[] OUI [] NON [] Je ne sais pas

J'apprends mieux en relisant des documents écrits sur une feuille plutôt que des documents sur la tablette.

[] OUI [] NON [] Je ne sais pas

Je comprends mieux en travaillant avec un stylo et un papier qu'avec une tablette.

[] OUI [] NON [] Je ne sais pas

Je comprends mieux en travaillant avec une tablette qu'avec un papier et un stylo.

[] OUI [] NON [] Je ne sais pas

\section{Présentation des résultats}

\subsection{Cohérence des résultats}

Une étude préliminaire des réponses aux quatre questions présentées ci-dessus a montré qu'un étudiant de première année et deux étudiants de deuxième année ont répondu NON à ces quatre questions tandis qu'un étudiant de première année et un étudiant de deuxième année ont répondu OUI à ces quatre questions. Etant donné que les réponses à ces quatre 
questions ne pouvaient pas être identiques si les étudiants avaient bien compris les énoncés, nous avons considéré que les réponses données par ces cinq étudiants ne pouvaient pas être considérées comme cohérentes et nous avons décidé de ne pas tenir compte de l'ensemble des réponses données par ces cinq étudiants.

Ainsi, notre enquête porte finalement uniquement sur les résultats de 42 (et pas 44) étudiants de première année universitaire et sur 34 (et pas 37) étudiants de deuxième année.

Sur ces 76 étudiants, quatre étudiants de première année et un étudiant de deuxième année ont répondu NON tandis que deux étudiants de première année ont répondu OUI aux deux premières ou au deux dernières questions précédemment citées. Ces réponses sont elles aussi incohérentes mais nous avons gardé les réponses de ces sept étudiants dans notre échantillon, étant donné que leurs réponses étaient cohérentes pour l'autre série de questions. Toutefois, il est important de garder en mémoire que les résultats qui seront donnés dans la suite de cette communication sous forme de pourcentages n'ont qu'une valeur indicative car au moins 7 étudiants sur 76 , soit $9 \%$ des étudiants, ont donné au moins une fois deux réponses incohérentes et car les réponses de l'ensemble des étudiants correspondent uniquement à des déclarations. Nous n'avons par exemple pas vérifié que tous les étudiants qui ont déclaré qu'ils avaient utilisé leur tablette pour prendre des photographies des montages réalisés pendant les travaux pratiques avaient réellement pris ces photos.

\subsection{Utilisation d'une tablette par les étudiants}

Etant donné que les énoncés des sujets de TP étaient lisibles par les étudiants uniquement sous format numérique, tous les étudiants ont déclaré avoir lu les énoncés des sujets sur leur tablette durant les TP d'électricité. De plus, d'après leurs déclarations, alors qu'il n'y avait eu aucune demande de l'enseignant pour utiliser la tablette, autre que celle demandant de lire les énoncés des sujets :

- $\quad 98 \%$ des étudiants de première année et $100 \%$ des étudiants de deuxième année ont pris avec leur tablette des photographies des montages réalisés, de l'écran de l'oscilloscope, du tableau ou des feuilles de son binôme (14\% des étudiants de première année et $9 \%$ des étudiants de deuxième année ont également déclaré avoir réalisé une vidéo ou un fichier son) (Figure 1);

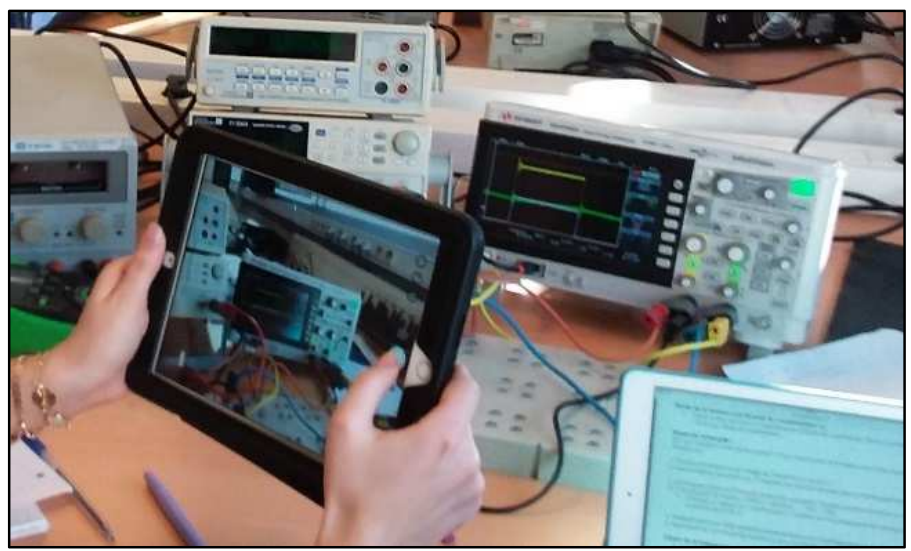

Figure 1. Etudiant prenant une photographie des résultats expérimentaux obtenus.

- $\quad 93 \%$ des étudiants de première année et $82 \%$ des étudiants de deuxième année ont utilisé leur tablette pour faire des calculs ou pour tracer un graphique ; 
- $\quad 62 \%$ des étudiants de première année et $41 \%$ des étudiants de deuxième année ont directement annoté avec leur tablette les photographies prises ou les énoncés des TP, afin de décrire les montages expérimentaux (Figure 2), les fonctionnalités des appareils utilisés (Figure 3 ) ou afin de noter par exemple des astuces expérimentales, des erreurs expérimentales à ne pas reproduire $(33 \%$ des étudiants de première année et $26 \%$ des étudiants de deuxième année ont écrit sur leur tablette dans un fichier texte annexe) ;

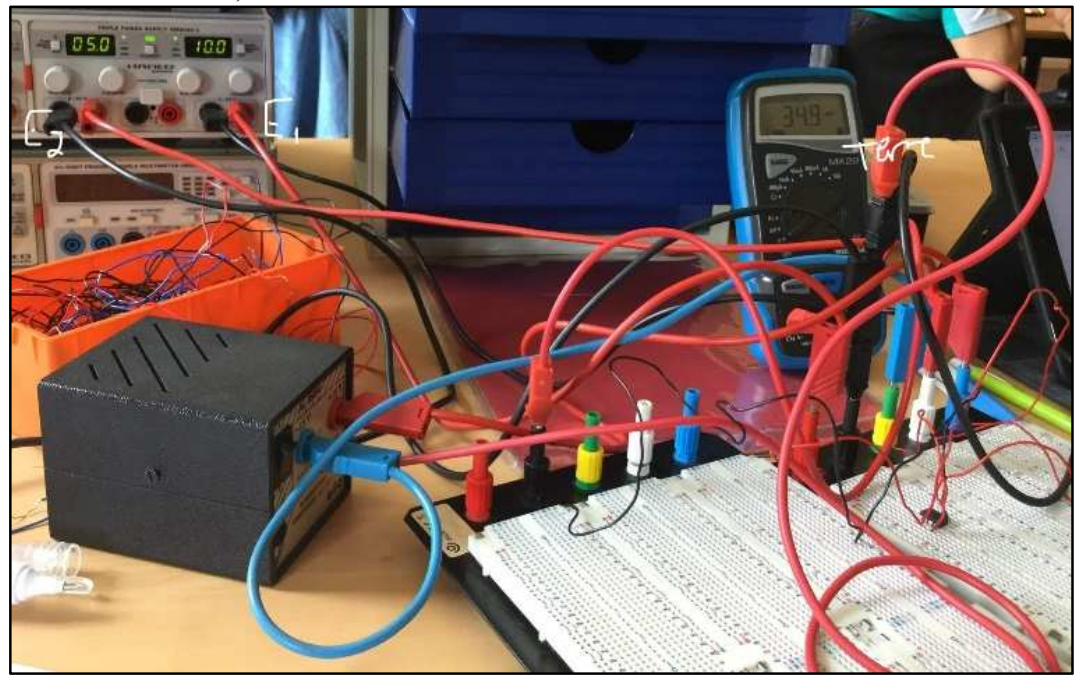

Figure 2. Annotations ( $E_{2}$ et Terre) d'une photographie d'un montage expérimental.



Figure 3. Annotations d'une photographie présentant les fonctionnalités d'un appareil.

- La tablette a servi à $92 \%$ des étudiants de première année et à $91 \%$ des étudiants de deuxième année pour partager des fichiers ou des informations avec leur binôme ou avec d'autres groupes de TP ;

- $\quad 78 \%$ des étudiants de première année et $94 \%$ des étudiants de deuxième année ont recherché des informations sur Internet (la tablette a aussi permis à $14 \%$ des 
étudiants de première année et à $21 \%$ des étudiants de deuxième année de poser des questions par mail au professeur entre les séances de TP);

- $\quad 55 \%$ des étudiants de première année et $69 \%$ des étudiants de deuxième année ont utilisé leur tablette pour se détendre pendant quelques minutes durant les TP en consultant leurs mails personnels, en allant sur Instagram...

Pour réviser les TP, toujours d'après les déclarations des étudiants :

- $\quad 88 \%$ des étudiants de première année et $100 \%$ des étudiants de deuxième année ont regardé à nouveau avec leur tablette les énoncés des TP, les graphiques, les annotations, les photographies, les vidéos et/ou les fichiers sons réalisés durant les TP ;

- $\quad 43 \%$ des étudiants de première année et $44 \%$ des étudiants de deuxième année ont ajouté des annotations supplémentaires grâce à leur tablette tandis que $26 \%$ des étudiants de première année et $29 \%$ des étudiants de deuxième année ont refait un ou plusieurs graphiques ;

- $\quad 67 \%$ des étudiants de première année et $65 \%$ des étudiants en deuxième année ont réalisé individuellement, avec leur binôme ou avec d'autres groupes de TP une fiche de révision sur leur tablette ;

- $\quad 71 \%$ des étudiants de première année et $91 \%$ des étudiants de deuxième année ont partagé des fichiers ou des informations lors des révisions grâce à leur tablette ;

- $\quad 64 \%$ des étudiants de première année et $91 \%$ des étudiants de deuxième année ont recherché des informations sur Internet avec leur tablette ;

- $\quad 29 \%$ des étudiants de première année et $18 \%$ des étudiants de deuxième année ont posé des questions par mail au professeur par l'intermédiaire de leur tablette ;

Enfin, $62 \%$ des étudiants de première année et $56 \%$ des étudiants de deuxième année ont utilisé leur tablette pour se détendre pendant quelques minutes durant les révisions en consultant leurs mails personnels, en allant sur Instagram, sur Youtube...etc.

\subsection{Apport de l'utilisation de la tablette pour les étudiants}

Concernant l'apport de l'utilisation de la tablette par les étudiants, même si $29 \%$ des étudiants de première et de deuxième années ont déclaré qu'ils auraient aussi bien appris et compris sans la tablette qu'avec la tablette, $74 \%$ des étudiants de première année et $68 \%$ des étudiants de deuxième année ont déclaré que l'utilisation de leur tablette les avait aidés lors des TP d'électricité. En effet, la tablette leur a permis :

- de rechercher facilement des informations sur Internet, pour $98 \%$ des étudiants de première année et $100 \%$ des étudiants de deuxième année interrogés ;

- de stocker et de retrouver plus facilement des documents, ce qui a été utile pour 95 $\%$ des étudiants de première année et $74 \%$ des étudiants de deuxième année ;

- de prendre des photographies pendant les TP, qui ont été utiles pour mieux réviser d'après $90 \%$ des étudiants de première année et $82 \%$ des étudiants de deuxième année ;

- d'annoter les photographies notamment et de relire ces annotations, afin de mieux comprendre et apprendre, pour $69 \%$ des étudiants de première année et $71 \%$ des étudiants de deuxième année ;

- de partager facilement des informations, des documents, etc. avec les autres étudiants, ce qui a permis de mieux comprendre et/ou apprendre pour $81 \%$ des étudiants de première année et $79 \%$ des étudiants de deuxième année ;

- d'utiliser un tableur-grapheur, qui trace plus rapidement des graphiques, ce qui a amélioré la compréhension pour $76 \%$ des étudiants de première et de deuxième années (seuls $24 \%$ des étudiants de première année et $41 \%$ des étudiants de 
deuxième année ont déclaré qu'ils comprenaient mieux en traçant les graphiques à la main sur une feuille de papier);

- de réaliser une vidéo ou un fichier son, ce qui a facilité la compréhension pour 33 $\%$ des étudiants de première année et pour $32 \%$ des étudiants de première année,

- de visionner des vidéos ou d'écouter des fichiers sons, ce qui a été utile pour mieux apprendre d'après $52 \%$ des étudiants de première année et $38 \%$ des étudiants de deuxième année ;

- de se détendre quelques minutes sur Internet avant de se remettre au travail, pour 64 $\%$ des étudiants de première année et $68 \%$ des étudiants de deuxième année (quelques étudiants ont toutefois été perturbés en utilisant la tablette car $12 \%$ d'entre eux en première année et $21 \%$ en deuxième année ont déclaré être tentés d'aller sur Internet au lieu de travailler tandis que $10 \%$ des étudiants de première année et $6 \%$ des étudiants de deuxième année ont considéré que l'utilisation de la tablette leur avait fait perdre du temps).

En revanche, il est plus simple pour $86 \%$ des étudiants de première année et pour $76 \%$ des étudiants de deuxième année d'écrire sur une feuille avec un stylo que dans un fichier texte avec la tablette. De plus, $81 \%$ des étudiants de première année et $79 \%$ des étudiants de deuxième année ont déclaré apprendre mieux en écrivant sur un papier plutôt que sur la tablette. Enfin, $55 \%$ des étudiants de première année et $59 \%$ des étudiants de deuxième année ont déclaré mieux comprendre ou mieux apprendre en travaillant avec un stylo et un papier qu'avec une tablette, tandis que $12 \%$ des étudiants de première année et $15 \%$ des étudiants de deuxième année ont déclaré le contraire.

\section{Interprétation des résultats}

\subsection{Comparaison des réponses des étudiants de première et de deuxième année}

La plupart des résultats concernant les étudiants de première et de deuxième année universitaire sont équivalents. Par exemple, $74 \%$ des étudiants de première année et $68 \%$ des étudiants de deuxième année ont déclaré que l'utilisation de leur tablette les avait aidés lors des TP d'électricité. Cette différence de $8 \%$ est non significative d'un point statistique, mais aussi parce que ces résultats correspondent uniquement à des déclarations dont nous avons montré précédemment que certaines étaient malheureusement incohérentes entre elles.

Seules quelques différences importantes peuvent être relevées : $62 \%$ des étudiants de première année mais uniquement $41 \%$ des étudiants de deuxième année ont directement annoté avec leur tablette les photographies prises ou les énoncés des TP tandis que $95 \%$ des étudiants de première année mais uniquement $74 \%$ des étudiants de deuxième année ont trouvé que la tablette était utile lors des TP d'électricité pour stocker et retrouver plus facilement des documents. Ces différences semblent principalement dues au fait que les annotations des étudiants portent principalement sur des consignes expérimentales à respecter, qui sont moins utiles car déjà davantage assimilées en deuxième année. De plus, peu d'étudiants de deuxième année feront à nouveau des TP d'électricité l'année suivante, contrairement aux étudiants de première année qui réaliseront tous des TP d'électricité en deuxième année, ce qui peut expliquer le fait que davantage d'étudiants de première année trouvent intéressant le fait de pouvoir stocker des documents pour les retrouver plus facilement l'année suivante. 
Une dernière différence existe : $91 \%$ des étudiants de deuxième année mais uniquement $71 \%$ des étudiants de première année ont partagé des fichiers ou des informations lors des révisions grâce à leur tablette. Cela peut être dû au fait que les étudiants de deuxième année se connaissaient mieux entre eux que ceux de première année et qu'ils partageaient donc plus facilement des informations ou au fait que les étudiants de deuxième année ont mieux compris l'intérêt d'un travail collaboratif par l'intermédiaire des tablettes que ceux de première année.

Ainsi, les quelques différences observées entre les réponses des étudiants de première et de deuxième année peuvent s'expliquer par des raisons didactiques : il semble donc que ce n'est pas l'effet de nouveauté qui a joué sur l'indice de satisfaction et sur la perception d'utilité de la tablette qu'avaient les étudiants. De même, les étudiants de première année ont semblé avoir une bonne maîtrise des tablettes six mois après les avoir reçues puisque leurs résultats diffèrent peu de ceux des étudiants de deuxième année, qui utilisent les tablettes depuis plus longtemps. Il sera donc possible dans la suite de cette étude de réaliser une interprétation générale des résultats, sans distinguer les réponses données par les étudiants de première ou de deuxième année.

\subsection{Interprétation générale des résultats}

Les résultats globaux de notre enquête montrent que l'utilisation d'une tablette a aidé environ $70 \%$ des étudiants de première et de deuxième année universitaire lors des TP d'électricité alors qu'il n'y a eu aucune demande de l'enseignant pour utiliser la tablette, autre que celle demandant de lire les énoncés des sujets. Ce résultat, assez important, doit être relativisé car il correspond à un impact perçu, déclaré, par les étudiants et non à un impact réel, mesuré dans le cadre d'une étude comparative. Par exemple, Clark [41] a montré que des étudiants pouvaient faire des témoignages très positifs alors que la pédagogie proposée ne leur avait en réalité pas permis de réaliser de progrès significatifs. Cependant, nous pouvons considérer que les étudiants, qui avaient tous reçu un Ipad au moins six mois auparavant pour leurs usages universitaires mais aussi personnels, se sont convenablement appropriés la tablette pour un usage éducatif et qu'ils n'ont pas eu besoin d'indications plus précises de la part du professeur pour s'en servir efficacement. La plupart d'entre eux ont pris des photographies ou ont réalisé des vidéos, les ont annotées, ont réalisé des graphiques, des calculs, ont communiqué entre eux ou avec le professeur, ont partagé des informations et ont créé des fiches de révision grâce à leur tablette.

En effet, d'autres formations ont eu lieu avant ces TP, pendant lesquelles une utilisation plus guidée des tablettes était demandée $[35,36]$, ce qui a permis aux étudiants de bien comprendre leur intérêt pédagogique. De plus, les conditions matérielles favorables, notamment un très bon accès au wifi durant les séances de $\mathrm{TP}$, ont permis à plus de quatre étudiants sur cinq de rechercher des informations scientifiques sur Internet durant les séances. Cela a aussi permis à plus de la moitié des étudiants de se détendre quelques minutes en allant surfer sur Internet pour leur usage personnel, sans que cela les empêche de travailler sérieusement en dehors de ces moments de détente (moins d'un étudiant sur cinq a déclaré être tenté d'aller sur Internet au lieu de travailler).

Ces conditions didactiques et matérielles favorables ont donc été positives pour l'apprentissage de l'électricité pour les étudiants, ce qui n'avait pas été le cas dans une autre étude portant sur l'utilisation des tablettes dans un lycée, avec des élèves un peu plus jeunes, pour qui la tablette était davantage une source de distraction [25]. Cette différence peut être due à la maturité supérieure des étudiants mais aussi au fait que les étudiants utilisent leur 
tablette quotidiennement, que ce soit en autonomie ou de façon plus guidée, alors que les lycéens l'utilisaient uniquement de façon plus épisodique. Comme cela a déjà été montré avec les boitiers de vote électroniques [42], l'utilisation intensive des tablettes permet donc une meilleure appropriation de celles-ci et augmente leur impact positif sur l'apprentissage [43]. Ainsi, il semble important de laisser les étudiants utiliser leur tablette lors de chaque séance d'enseignement, que ce soit en autonomie ou dans le cadre d'une utilisation de la tablette plus guidée, afin qu'ils s'approprient et comprennent mieux les nombreux avantages mais aussi les inconvénients que peut leur apporter l'utilisation d'une tablette et qu'ils soient capables de gérer les nombreuses sollicitations extrascolaires induites par l'utilisation d'un tel outil connecté.

Concernant l'utilisation en autonomie des tablettes, celle-ci semble être intéressante si elle est couplée avec une utilisation plus guidée dans d'autres séances d'enseignement, au moins lors des premiers mois d'utilisation, car elle permet à chaque étudiant d'apprendre à utiliser les différentes fonctionnalités de la tablette puis de choisir celles qui l'intéressent et qui lui sont utiles personnellement dans son apprentissage. Cette utilisation non prescriptive favorise également l'échange et le partage de données entre les étudiants et également quelquefois avec le professeur. Cependant, cela semble cependant plus difficile à mettre en œuvre avec des élèves plus jeunes, pour des difficultés de gestion de classe notamment [25, 28], alors que c'est justement le fait de pouvoir l'utiliser en autonomie, en complément d'une utilisation plus guidée, qui semble augmenter leur utilité de façon générale. En effet, si elles ne remplacent pas encore les ordinateurs pour certaines expérimentations (EXAO) ou pour la programmation d'algorithmes complexes, les tablettes permettent d'effectuer très facilement de nombreuses tâches comme la prise de photographies d'expériences et leur annotation ou la réalisation de vidéos, ainsi que le partage d'informations, qui sont très utiles aujourd'hui à la plupart des étudiants. Cela favorise leur activité durant les séances d'enseignement, ce qui est un des leviers pour améliorer l'apprentissage [44] et cela oblige les étudiants à avoir une analyse réflexive des TP qu'ils sont en train d'effectuer afin de savoir ce qu'il est important de photographier, de filmer et/ou de partager.

\section{Conclusion et perspectives de recherche}

En conclusion, les résultats de cette étude montrent un impact positif de l'utilisation des tablettes en autonomie lors des TP d'électricité, aussi bien en première qu'en deuxième année universitaire, cet impact positif semblant notamment être dû à la maturité des étudiants ainsi qu'au fait que des séances plus guidées d'utilisation des tablettes avaient été proposées précédemment. Ces résultats sont cependant encore très généraux et ils ne prennent notamment pas en compte les différents profils des étudiants par rapport à leurs usages personnels d'une tablette lors de séances d'enseignement. L'objectif est donc de continuer à étudier cette utilisation en effectuant une analyse plus fine, personnalisée de l'utilisation d'une tablette par chaque étudiant par rapport à leur distance CPC (connaissance / pratique / conviction) avec l'utilisation d'une tablette, dépendant des connaissances de l'étudiant, de ses pratiques d'apprentissage et de ses convictions sur la "bonne" manière d'apprendre [45, 46]. Enfin, nous essaierons de généraliser ces résultats dans un cadre plus large que celui des TP d'électricité à l'Université en travaillant également sur les séances plus guidées demandant explicitement l'utilisation d'une tablette numérique tactile.

\section{Remerciements}

Je tiens à remercier chaleureusement Vincent Villar, qui a fait passer les questionnaires aux étudiants. 


\section{Bibliographie}

1. M. Archambault. Apprendre et enseigner avec une tablette : une expérimentation au lycée (2015). Accédé le 15 mars 2021 : http://www.uoh.fr/front/notice?id=133c9a2c$\underline{35 \mathrm{eb}-4754-\mathrm{acaf}-61466 \mathrm{ec} 775 \mathrm{a} 9}$

2. T. Armstrong. The Multiple Intelligences of Reading and Writing Making the Words Come Alive. ASCD (2003).

3. J. Belleau. Les intelligences multiples. Collection orientante, fascicule de l'élève et fascicule d'accompagnement, Éditions Septembre (2003).

4. N. Brassard. Pourquoi varier les approches pédagogiques ? Le tableau, n ${ }^{\circ} 1$ (2012). Accédé le le 15 mars 2021 : http://pedagogie.uquebec.ca/le-tableau/pourquoi-varier-lesapproches-pedagogiques

5. L. Campbell,.B Campbell, \& D. Dickinson. Teaching and Learning Through Multiple Intelligences. Allyn and Bacon (2003).

6. CNESCO. Rapport du jury de la conférence de consensus Cnesco-Ifé/ENS de Lyon sur la différenciation pédagogique (2017). Accédé le 15 mars 2021 : http://www.cnesco.fr/fr/differenciation-pedagogique-decouvrez-les-recommandationsdu-jury/

7. N. Coppens, G. Rebmann \& V. Munier. Les TICE : une passerelle entre la recherche en éducation et l'enseignement des sciences? Exemple d'usages en classe d'exercices informatisés permettant de suivre les conceptions de lycéens français en mécanique. In Actes du congrès 2011 de l'Association Francophone Internationale de Recherche Scientifique en Éducation (AFIRSE) "La recherche en éducation dans le monde, où en sommes-nous? Thèmes, méthodologies et politiques de recherche", 14-17 juin 2011, Paris-UNESCO (2011).

8. N. Coppens, G. Rebmann, \& V. Munier. Suivre l'évolution des conceptions des élèves en mécanique : développement et évaluation d'exercices informatisés. Didaskalia, 35, pp. 37-58 (2009). Accédé le 15 mars 2021 : http://documents.irevues.inist.fr/bitstream/handle/2042/31136/DIDASKALIA2009_35 37.pdf? sequence $=1$

9. A. Daele. Différencier son enseignement à l'université ? Pédagogie universitaire Enseigner et Apprendre en Enseignement Supérieur (2009). Accédé le 15 mars 2021 : https://pedagogieuniversitaire.wordpress.com/2009/05/31/differencier-sonenseignement-a-luniversite/

10. A. Feyfant. La différenciation pédagogique en classe. Dossier de veille de l'IFÉ, 113 (2016). Accédé le 15 mars 2021 : http://ife.enslyon.fr/vst/DA/detailsDossier.php?parent=accueil\&dossier=113\&lang=fr

11. H. E. Gardner. Multiple Intelligences: New Horizons in Theory and Practice. Hachette UK (2006).

12. GRIESP. Réussir en mécanique du cycle 3 au cycle terminal du lycée (2018). Accédé le 15 mars 2021 : http://eduscol.education.fr/cid129214/recherche-et-innovation-enphysique-chimie.html

13. P. Perrenoud. Pédagogie différenciée. Des intentions à l'action. Issy-les-Moulineaux, ESF (1997).

14. H. Przesmycki. La pédagogie différenciée. Paris: Hachette education (2004).

15. Service de soutien à la formation de l'Université de Sherbrooke. Différenciation pédagogique

(2015)

Accédé

le

15 mars 2021 
https://www.usherbrooke.ca/ssf/veille/perspectives-ssf/numeros-precedents/decembre2015/le-fin-mot-differenciation-pedagogique/

16. N. Tardif. La pédagogie différenciée au service de l'apprentissage, Vie pédagogique, 134, pp. 21-24 (2005). Accédé le 15 mars 2021 : https://www4.ac-nancy-metz.fr/ia54circos/ientoul/sites/ientoul/IMG/pdf/differencier.pdf

17. C. Vaufrey. Pédagogie différenciée à l'université : lancez-vous ! Quelques manières simples d'introduire de la diversité dans son enseignement universitaire, sans réinventer son métier (2017). Accédé le 15 mars 2021 : https://cursus.edu/articles/24827

18. J.-M. Zakhartchouk. Au risque de la pédagogie différenciée. Paris : Institut National de Recherche Pédagogique (2001).

19. C. F. Mang \& L. J. Wardley. Student Perceptions of Using Tablet Technology in PostSecondary Classes / Perceptions des étudiants quant à l'utilisation des tablettes électroniques dans les classes universitaires. Canadian Journal of Learning and Technology, 39 (2013). Accédé le 15 mars 2021 : https://www.cjlt.ca/index.php/cjlt/article/view/26299

20. Ministère de l'Education Nationale française. Tablettes tactiles : retours d'expérimentations et potentialités pédagogiques (2016). Accédé le 15 mars 2021 : http://eduscol.education.fr/cid71927/tablettes-tactiles-retours-d-experimentations-etpotentialites-pedagogiques.html

21. J. E. Anderson, P. H. Schwager \& R. L. Kerns. The Drivers for Acceptance of Tablet PCs by Faculty in a College of Business, Journal of Information Systems Education, 17 / 4, pp. 429-440 (2006). Accédé le 15 mars 2021 : http://www.paulschwager.com/pubs/2006JISE.pdf

22. B. Clarke \& S. Svanaes. One-to-one tablets in secondary schools: an evaluation study, stage 1 : 2011-2012. Londres, Family Kids and Youth (2012). Accédé le 15 mars 2021 : $\quad$ http://www.kidsandyouth.com/pdf/FK\%26Y\%20T4S\%20Stage\%201\%202011$12 \% 20$ Report.pdf

23. H. Dundar \& M. Akçayır. Implementing tablet PCs in schools: Students' attitudes and opinions, Computers in Human Behavior, 32, pp. 40-46 (2014). Accédé le le 15 mars 2021 : https://www.sciencedirect.com/science/article/pii/S074756321300441X

24. T. Karsenti \& A. Fievez. L'IPad à l'école : usages, avantages et défis : résultats d'une enquête auprès de 6057 élèves et 302 enseignants du Québec (Canada), Montréal, CRIFPE (2013). Accédé le 15 mars 2021 : https://inshea.fr/sites/default/files/fichierorna/rapport_iPad_Karsenti-Fievez_FR.pdf

25. M. G. Landau. Les tablettes numériques à l'école. De quelle manière les différents acteurs perçoivent et utilisent cette technologie dans l'enseignement secondaire? Un étude de cas dans un lycée de Monaco. Mémoire de Master, Université Paul Valéry Montpellier III (2014). Accédé le 15 mars 2021 : http://espeformation.unistra.fr/webdocs/tablettes/documents/Memoire tablettes.pdf

26. Ministère de l'Education Nationale française. Tablettes tactiles et enseignement (2015). $\begin{array}{lllll}\text { Accédé } & \text { le } & 15 & \text { mars } & \end{array}$ http://eduscol.education.fr/numerique/dossier/apprendre/tablettetactile/@@document_whole

27. N. Zaugra. 50 lycées du Grand-Est abandonnent manuels scolaires pour tablettes et wifi. Lorraineactu (25 octobre 2017). Accédé le 15 mars 2021 : https://actu.fr/societe/50lycees-grand-est-abandonnent-manuels-scolaires-tablettes-wifi_12988461.html

28. L. Boujol. Usage pédagogique des tablettes tactiles numériques : une étude de cas en enseignement primaire genevois. Genève : Université de Genève, TECFA/MALTT, 
Faculté de Psychologie et des Sciences de l'Éducation (2014). Accédé le 15 mars 2021 $:$ http://tecfa.unige.ch/tecfa/maltt/memoire/boujol2014.pdf

29. OCDE. Connectés pour apprendre? Les élèves et les nouvelles technologies (2015). Accédé le 15 mars 2021 : http://www.oecd.org/fr/education/scolaire/Connectes-pourapprendre-les-eleves-et-les-nouvelles-technologies-principaux-resultats.pdf

30. M. Prensky. Digital natives, digital immigrants. On the Horizon, 9/5 (2001). Accédé le 15 mars 2021 : https://www.marcprensky.com/writing/Prensky\%20\%20Digital\%20Natives, \%20Digital\%20Immigrants\%20-\%20Part1.pdf

31. N. Guichon. Les usages des TIC par les lycéens - déconnexion entre usages personnels et usages scolaires. Sciences et Technologies de l'Information et de la Communication pour l'Éducation et la Formation, ATIEF, 19 (2012). Accédé le 15 mars 2021 : https://hal.archives-ouvertes.fr/hal-00806411

32. J. Boissière, S. Fau et F. Pedró. Le numérique : Une chance pour l'école. Paris, Armand Colin (2013).

33. C. F. Mang \& L. J. Wardley. Effective adoption of tablets in post-secondary education: recommendations based on a trial of iPads in university classes. Journal of Information Technology Education: Innovations in Practice, 11, pp. 301-317 (2012). Accédé le 15 mars 2021 : http://www.jite.org/documents/Vol11/JITEv11IIPp301-317Mang1138.pdf

34. S. Bégin. Prix d'excellence pour le CPI de l'ECPM. ECPM Infos, 17 (2016). Accédé le 15 mars 2021 : https://ecpm.unistra.fr/uploads/media/ECPMinfos-N17-mai-2016.pdf

35. S. Despax, P. Bernard \& V. Villar. Les «VIDEXOS » du cycle préparatoire intégré de l'Université de Strasbourg : comment développer ses compétences scientifiques en premier cycle universitaire grâce à l'iPad ? Communication dans un congrès, $4^{e ̀ m e}$ colloque international en éducation, 18 et 19 mai 2017, Montréal, Canada (2017).

36. V. Villar, P. Bernard \& S. Despax. Les Videxos. L'actualité chimique, 415, (2017). Accédé le 15 mars 2021 : http://www.lactualitechimique.org/Les-VIDEXOS

37. N. Coppens. Analyse de l'utilisation en autonomie de tablettes numériques tactiles par des étudiants lors de Travaux Pratiques d'électricité à l'Université. In Actes du $6^{\text {ème }}$ colloque international en éducation "Enjeux actuels et futurs de la formation et de la profession enseignante", 25-26 avril 2019, Montréal, Canada (2019).

38. B. Drot-Delange \& M. More. Attitudes envers l'informatique des élèves de terminale scientifique. Quelques résultats exploratoires. Communication dans un congrès, Sciences et technologies de l'information et de la communication (STIC) en milieu éducatif, Clermont-Ferrand, France (2013). Accédé le 15 mars 2021 : https://halshs.archives-ouvertes.fr/edutice-00877150/

39. F. D. Davis. User acceptance of information technology: system characteristics, user perceptions and behavioral impacts, International Journal of Man-Machine Studies, 38/3, pp. 475-487 (1993). Accédé le 15 mars 2021 : https://www.sciencedirect.com/science/article/pii/S0020737383710229

40. J. P. Rossing, W. M. Miller, A. K. Cecil \& S. E. Stamper. iLearning: The future ofhigher education? Student perceptions on learning with mobile tablets. Journal of the Scholarship of Teaching and Learning, 12(2), pp. 1-26 (2012). Accédé le 15 mars 2021 : https://files.eric.ed.gov/fulltext/EJ978904.pdf

41. R. E. Clark. Antagonism between achievement and enjoyment in ATI studies. Educational Psychologist, 17, pp. 92-101 (1982). Accédé le 15 mars 2021 : https://www.tandfonline.com/doi/abs/10.1080/00461528209529247

42. N. Coppens \& V. Villar. Comparaison de deux types d'utilisation, ponctuelle ou intensive, d'un même dispositif informatisé d'évaluation : les boitiers de vote 
électronique. In Actes du $26^{\text {ème }}$ colloque international de l'Association pour le Développement des Méthodologies d'évaluation en éducation (ADMEE-Europe) "Cultures et politiques de l'évaluation en éducation et en formation", 15-17 janvier 2014, Marrakech, Maroc (2014). Accédé le 15 mars 2021 : http://maarifcentre.org/admee2014/images/actes/coppens-nicolas-actes2014.pdf

43. M. Lebrun. Impacts des TIC sur la qualité des apprentissages des étudiants et le développement professionnel des enseignants : vers une approche systémique. Sciences et Technologies de l'Information et de la Communication pour l'Éducation et la Formation, ATIEF, 18 (2011). Accédé le 15 mars 2021 : https://halshs.archivesouvertes.fr/hal-00696443/

44. M. Poumay. Six leviers pour améliorer l'apprentissage des étudiants du supérieur, Revue internationale de pédagogie de l'enseignement supérieur, 30-1 (2014). Accédé le 15 mars 2021 : http://journals.openedition.org/ripes/778

45. N. Coppens. Le suivi des conceptions des lycéens en mécanique : développement et usages d'exercices informatisés. Université Paris-Diderot - Paris 7 (2007). Accédé le 15 mars $2021:$ https://tel.archives-ouvertes.fr/tel-00203891

46. G. Rebmann. Investigation of actual use of informatic tools by science teachers: the French case. In European Commission - Science Teacher Training in an Information Society (STTIS), F. Stylianidou, J. Ogborn, O. Andresen, E. Balzano, G. Giberti, R. Guttiérrez, S. Dankert Kolsto, G. Monroy, O. Pérez, R. Pinto, A. Quale, G. Rebmann \& E. Sassi, The nature of use by science teachers of informatic tools, Tranversal Report on WP1.2, pp. 88-102 (2000). Accédé le 15 mars 2021 : https://www.researchgate.net/profile/Emilio-

Balzano/publication/258275752 The nature of use by science teachers of informat ic tools/links/0c960527a913e51e85000000/The-nature-of-use-by-science-teachers-ofinformatic-tools.pdf 